\title{
International Guidelines for Pediatric Radiopharmaceutical Administered Activities
}

$\mathbf{P}$ ediatric applications of nuclear medicine provide invaluable information in the diagnosis and follow-up of many disorders. However, radiopharmaceutical administered activities in children have not been standardized in North America until recently.

Over the years, individual groups and institutions have developed their own approaches toward determining pediatric doses. In the past, determination of pediatric radiopharmaceutical activities relied on several factors, including the practitioner's experience and preference, type of study, available instrumentation, patient size, photon flux, examination time, experience in dealing with children, and patient's ability to cooperate. As a consequence of the lack of standards, administered activities of pediatric radiopharmaceuticals have varied quite broadly. It follows that the resultant radiation exposure to children undergoing nuclear medicine examinations also has varied quite broadly. Higher amounts of activity may not result in improved diagnostic sensitivity or accuracy, and low doses that do not permit an adequate examination should be considered unnecessary radiation exposure and therefore avoided (1).

To date, there have been no documented risks to the patient with the low doses of radiation from nuclear medicine studies (2). However, it is considered prudent and good practice to perform these studies using the lowest amount of radioactivity that will also ensure that the procedure has diagnostic value.

A survey of specialized pediatric hospitals in North America revealed a rather large variation in individual protocols resulting in a 3- to 10-fold range of administered activities among children over the age of $1 \mathrm{y}$. Importantly, a 10- to 20 -fold variation in administered activities was found in the youngest children (3).

In recent years, there has been increasing concern about potential risks to children from the use of diagnostic imaging that applies ionizing radiation $(4,5)$. Coinciding with this concern, the level of interest and cooperation among pediatric nuclear medicine experts in the development of guidelines for the administered activity of pediatric radiopharmaceuticals has been unprecedented, and their efforts have been fruitful.

From 2008 through 2013, several expert consensus symposiums and workshops addressing administered doses of pediatric radiopharmaceuticals have been conducted during annual meetings of the Society of Nuclear Medicine and Molecular Imaging (SNMMI), the Society for Pediatric Radiology (with the auspices of the Image Gently Campaign), and the European Association of Nuclear Medicine (EANM).

Received Mar. 19, 2014; accepted Mar. 19, 2014

For correspondence or reprints contact: S. Ted Treves, Harvard Medical School, Division of Nuclear Medicine and Molecular Imaging, Brigham and Women's Hospital, 75 Francis St., Boston, MA 02115.

E-mail: streves@partners.org

Published online Apr. 17, 2014.

COPYRIGHT (C) 2014 by the Society of Nuclear Medicine and Molecular Imaging, Inc.

DOI: $10.2967 /$ jnumed.114.139980
In Europe, early recommendations for dosing radiopharmaceuticals in children and adolescents were published in 1990 (6). In 2007, a new and completely revised version of the EANM dosage card was developed by the EANM Dosimetry Committee and the EANM Paediatrics Committee $(7,8)$.

In North America, the Image Gently Campaign encouraged the formation of an expert group to overcome the lack of pediatric guidelines and to look into the possibility of developing pediatric consensus guidelines. Early efforts resulted in the development and publication of the 2010 North American Pediatric Administered Radiopharmaceutical Dose Guidelines. These guidelines were approved by the SNMMI, the Society for Pediatric Radiology, and the American College of Radiology $(9,10)$.

Examination of the 2010 North American guidelines and the 2007 EANM dosage card revealed agreements and discrepancies between their respective recommended administered activities. This discovery provided an impetus to evaluate whether a closer agreement between the two guidelines could be achieved. During the 2012 and 2013 EANM annual congresses, a working group including members of both the EANM and the SNMMI met to study the possibility of harmonizing the guidelines published by the two societies.

Although the North American and EANM guidelines use different models, these meetings have culminated in the development of a set of international guidelines, also referred to as "Pediatric Radiopharmaceutical Administration: Harmonization Guidelines." Twelve radiopharmaceuticals are included in the new guidelines, and others will soon be incorporated. A modified version of the EANM dosage card incorporating the suggested changes is now available online (11).

These guidelines are meant to be just that and are not meant to dictate or regulate individual practices. Appropriate selection of the administered radiopharmaceutical activity should relate to the patient population, available equipment, specific clinical requirements, and physician's judgment. Therefore, deviation from the administered activities listed in these guidelines should be considered appropriate when clinically indicated. Individual practitioners may use a lower administered activity if their equipment or software permits them to do so (12-14). Under special circumstances, higher administered activities may be required in certain patients under the direction of the nuclear medicine physician $(1,15)$.

As new technologies and applications of nuclear medicine evolve, these groups should continue to meet to refine and update the guidelines. Because data on the biokinetics and dosimetry of commonly used radiopharmaceuticals in pediatric nuclear medicine are missing, we should focus in the next few years on obtaining more and better data on image quality, biokinetics, and dosimetry as a basis for further improving the recommendations for administered activities $(16,17)$.

This evolved approach should hopefully lead to a broad optimization of pediatric radiopharmaceutical administered activities. 
Application of the guidelines will allow many pediatric nuclear medicine patients to receive radiopharmaceutical doses lower than those traditionally given, resulting in an overall reduction of radiation exposure in these patients.

We hope that the release of these new harmonized dosage recommendations will bring about a higher level of unification within the practice of pediatric nuclear medicine around the globe.

\section{ACKNOWLEDGMENTS}

The Pediatric Dosage Harmonization Working Group members and contributors include Ronald Boellaard (EANM Physics Committee), Wesley Bolch (SNMMI), George Sgouros (SNMMI), Lise Borgwardt (EANM Pediatrics Committee), Arturo Chiti (EANM), Frederic Fahey (SNMMI), Michael Gelfand (SNMMI and Image Gently), Michael Lassmann (EANM Dosimetry Committee), Marguerite Parisi (Society for Pediatric Radiology, Nuclear Medicine Committee and Image Gently), Thomas Pfluger (EANM Pediatrics Committee), Stephanie Spottswood (SNMMI, Pediatric Imaging Council), and S. Ted Treves (SNMMI and Image Gently).

\section{REFERENCES}

1. Treves ST, Baker A, Fahey FH, et al. Nuclear medicine in the first year of life. J Nucl Med. 2011;52:905-925.

2. Ernst M, Freed ME, Zametkin AJ. Health hazards of radiation exposure in the context of brain imaging research: special consideration for children. $\mathrm{J} \mathrm{Nucl}$ Med. 1998;39:689-698.

3. Treves ST, Davis RT, Fahey FH. Administered radiopharmaceutical doses in children: a survey of 13 pediatric hospitals in North America. J Nucl Med. 2008;49: 1024-1027.

4. Brenner D, Elliston C, Hall E, Berdon W. Estimated risks of radiation-induced fatal cancer from pediatric CT. AJR. 2001;176:289-296.

5. Hall P, Adami HO, Trichopoulos D, et al. Effect of low doses of ionising radiation in infancy on cognitive function in adulthood: Swedish population based cohort study. BMJ. 2004;328:19.
6. Piepsz A, Hahn K, Roca I, et al. A radiopharmaceutical schedule for imaging in paediatrics. Eur J Nucl Med. 1990;17:127-129.

7. Lassmann M, Biassoni L, Monsieurs M, Franzius C, Jacobs F. EANM Dosimetry and Paediatrics Committees. The new EANM paediatric dosage card. Eur J Nucl Med Mol Imaging. 2007;34:796-798.

8. Lassmann M, Biassoni L, Monsieurs M, Franzius C. The new EANM paediatric dosage card: additional notes with respect to F-18. Eur J Nucl Med Mol Imaging. 2008;35:1666-1668.

9. Gelfand MJ, Parisi MT, Treves ST. Pediatric radiopharmaceutical administered doses: 2010 North American consensus guidelines. J Nucl Med. 2011;52:318322.

10. Treves ST, Parisi MT, Gelfand MJ. Pediatric radiopharmaceutical doses: new guidelines. Radiology. 2011;261:347-349.

11. Lassmann M, Treves ST. Paediatric radiopharmaceutical administration: harmonization of the 2007 EANM paediatric dosage card (version 1.5.2008) and the 2010 North American consensus guidelines. Eur J Nucl Med Mol Imaging. March 6, 2014 [Epub ahead of print].

12. Sheehy N, Tetrault TA, Zurakowski D, Vija AH, Fahey FH, Treves ST. Pediatric ${ }^{99 \mathrm{~m}}$ Tc-DMSA SPECT performed by using iterative reconstruction with isotropic resolution recovery: improved image quality and reduced radiopharmaceutical activity. Radiology. 2009;251:511-516.

13. Stansfield EC, Sheehy N, Zurakowski D, Vija AH, Fahey FH, Treves ST. Pediatric ${ }^{99 m}$ Tc-MDP bone SPECT with ordered subset expectation maximization iterative reconstruction with isotropic 3D resolution recovery. Radiology. 2010;257:793-801.

14. Hsiao EM, Cao X, Zurakowski D, et al. Reduction in radiation dose in mercaptoacetyltriglycerine renography with enhanced planar processing. Radiology. 2011;261:907-915.

15. Treves ST, Fahey F. Pediatric nuclear medicine and radiation dose. Semin Nucl Med. In press.

16. Eberlein U, Broer JH, Vandevoorde C, et al. Biokinetics and dosimetry of commonly used radiopharmaceuticals in diagnostic nuclear medicine: a review. Eur J Nucl Med Mol Imaging. 2011;38:2269-2281.

17. Sgouros G, Frey EC, Bolch WE, Wayson MB, Abadia AF, Treves ST. An approach for balancing diagnostic image quality with cancer risk: application to pediatric diagnostic imaging of 99mTc-DMSA. J Nucl Med. 2011;52: 1923-1929.

S. Ted Treves and Michael Lassmann
for the EANM/SNMMI Pediatric Dosage
Harmonization Working Group 